\title{
MULTI-SENSOR IMAGE REGISTRATION BASED-ON LOCAL PHASE COHERENCE
}

\author{
Rania Hassen, Zhou Wang and Magdy Salama \\ Department of Electrical and Computer Engineering \\ University of Waterloo, Waterloo, Ontario, N2L 3G1, Canada \\ raniahassen@ieee.org, zhouwang@ieee.org,m.salama@ece.uwaterloo.ca
}

\begin{abstract}
The major challenges in automatic multi-sensor image registration are the inconsistency in intensity or contrast patterns, and the existence of partial or missing information between images. Here we propose a novel image registration method based on local phase coherence features, which are insensitive to changes in intensity or contrast. Furthermore, a new objective function based on weighted mutual information is proposed, where less weight is given to the objects that have no correspondence between images. The proposed method has been tested on both synthetic and medical images and evaluated based on registration accuracy. Our experiments demonstrate good performance of the proposed approach with missing or partial data, with significant changes in contrast, and with the presence of noise.
\end{abstract}

Index Terms - image registration, local phase coherence, mutual information, weighted mutual information

\section{INTRODUCTION}

Multi-sensor image registration is an active research problem that has a variety of applications in medical image analysis, remote sensing and computer vision. The main goal is to geometrically align images of the same scene taken by different sensors or modalities. Challenging issues found in multi-sensor images include: (1) the relationship is unknown between intensity values of corresponding pixels; (2) Image contrast may differ in the same regions from each other; (3) Multiple intensity values in one image may map to a single intensity value in another image; (4) Structures present in one image may not appear in another image.

Image registration methods may be categorized into intensitybased and feature-based approaches. Intensity-based approaches operate under the assumption that a strong relationship exists between intensities of images to be registered. They attempt to match intensity values by optimizing certain objective function such as the mutual information (MI) between two images $[1,2]$. In multi-sensor image registration, however, it is sometimes difficult to find a reliable relationship. Contrast and brightness variation between images is another challenge, which often leads to poor registration performance. Feature-based approaches do not work directly with pixel values, but match higher level features such as edges or corners [3]. This makes them more robust to intensity variations and sensor noise that arise in multi-sensor registration.

The performance of feature-based methods depends highly on the features being used. In this paper, we proposed a novel type of feature extraction method based on local phase coherence [4] measurement. This feature describes the behavior of local phase structure at different scales in the vicinity of sharp image features [4]. We show that local phase coherence measurement is insensitive to noise as well as intensity and contrast variations. It thus provides an effective and robust approach to capture salient image features in multi-senor images. In the literature, local phase has been used in a number of machine vision and image processing applications, such as estimation of image motion [5] and disparity [6], description of image textures [7], and recognition of persons using iris patterns [8]. In [9], local monogenic phase was employed directly for computing the geometric registration parameters, where phase singularities could make the algorithm sensitive to input scale perturbations, small changes in spatial position and noise [10]. In [11], phase congruency representation [12] was used to align the images on a pixel level followed by refinement to a sub-pixel level. The local phase coherence model employed in this paper is more general than phase congruency since the latter describes phase alignment at only the exact location rather than the vicinity of image features.

\section{LOCAL PHASE COHERENCE}

We will first introduce the concept of local phase coherence in 1D, which can be easily generalized to high dimensions. Given a signal $f(x)$ localized near the position $x_{0}$ where $f(x)=f_{0}\left(x-x_{0}\right)$, its wavelet transform can be written as:

$$
F(s, p)=\int f(x) w_{s, p}^{*}(x) d x=\left[f(x) * \frac{1}{\sqrt{s}} g\left(\frac{x}{s}\right) e^{\jmath \omega_{c} x / s}\right]_{x=p}
$$

where $s \in R^{+}$is the scale factor, $p \in R$ is the translation factor and the family of wavelets $w_{s, p}(x)$ is derived from the mother wavelet $w(x)=g(x) e^{j \omega_{c} x}$ (with center frequency $\omega_{c}$ and a slowly varying and symmetric envelop function $g(x)$ ):

$$
w_{s, p}(x)=\frac{1}{\sqrt{s}} w\left(\frac{x-p}{s}\right)=\frac{1}{\sqrt{s}} g\left(\frac{x-p}{s}\right) e^{\jmath \omega_{c}(x-p) / s} .
$$

Using the convolution theorem, and the shifting and scaling properties of Fourier transform, Eq. (1) can be written as:

$$
\begin{aligned}
F(s, p) & =\frac{1}{2 \pi} \int_{-\infty}^{\infty} F(\omega) \sqrt{s} G\left(s \omega-\omega_{c}\right) e^{j \omega p} d \omega \\
& =\frac{1}{2 \pi} \int_{-\infty}^{\infty} F_{0}(\omega) \sqrt{s} G\left(s \omega-\omega_{c}\right) e^{j \omega\left(p-x_{0}\right)} d \omega \\
& =\frac{1}{2 \pi \sqrt{s}} \int_{-\infty}^{\infty} F_{0}\left(\frac{\omega}{s}\right) G\left(\omega-\omega_{c}\right) e^{j \omega\left(p-x_{0}\right) / s} d \omega
\end{aligned}
$$

where $F(\omega), F_{0}(\omega)$ and $G(\omega)$ are the Fourier transforms of $f(x)$, $f_{0}(x)$ and $g(x)$, respectively. From Eq. (3), we can see that the phase of $F(s, p)$ depends on the nature of $F_{0}(\omega)$. If $F_{0}(\omega)$ is scale invariant, meaning that $F_{0}(\omega / s)=K(s) F_{0}(\omega)$, where $K(s)$ is a 


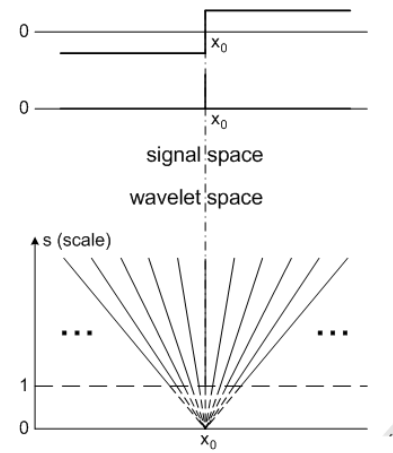

(a)

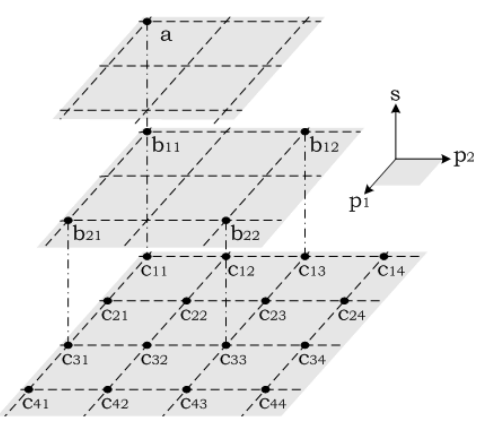

(b)
Fig. 1. (a) Local phase coherence of localized sharp feature at $x_{0}$; (b) $2 \mathrm{D}$ sampling grid of wavelet coefficients.

real function of only $s$, but independent of $\omega$, then from Eq. (3) we have:

$$
F(s, p)=\frac{K(s)}{\sqrt{s}} F\left(1, x_{0}+\frac{p-x_{0}}{s}\right) .
$$

Because $K(s)$ and $s$ are both real, we obtain:

$$
\Phi(F(s, p))=\Phi\left(F\left(1, x_{0}+\frac{p-x_{0}}{s}\right)\right) .
$$

Eq. (5) suggests a strong phase coherence relationship across scale and space, where equal phase contours in the $(s, p)$ plane form straight lines that converge exactly at the location of the feature $x_{0}$, as illustrated in Fig. 1(a). More generally, the phase at any given scale may be computed from the phase at any other scale by simply rescaling the position axis.

The above results can be extended for two-dimensional signals or images [4], where the phases of complex wavelet coefficients exhibit a consistent relationship between coefficients at adjacent scales in the vicinity of sharp image features, such as edges and lines. Therefore, the fine-scale coefficients can be well predicted from their coarser-scale coefficients, provided that the local phase satisfies the phase coherence relationship defined in Eq. (5). In the case that the positions of the neighboring complex wavelet coefficients are aligned as in Fig. 1(b), the phase prediction expression from coarser scale coefficients $\left\{a, b_{11}, b_{12}, b_{21}, b_{22}\right\}$ to the finest scale coefficients $\left\{c_{i j}\right\}$ is given by:

$$
\begin{aligned}
& \hat{\Phi}\left(\left\{c_{i j}\right\}\right)= \\
& \Phi\left(\left(a^{*}\right)^{2} \cdot\left[\begin{array}{llll}
b_{11}^{3} & b_{11}^{2} b_{12} & b_{11} b_{12}^{2} & b_{12}^{3} \\
b_{11}^{2} b_{21} & b_{11}^{2} b_{22} & b_{11} b_{12} b_{22} & b_{12}^{2} b_{22} \\
b_{11} b_{21}^{2} & b_{11} b_{21} b_{22} & b_{11} b_{22}^{2} & b_{12} b_{22}^{2} \\
b_{21}^{3} & b_{21}^{2} b_{22} & b_{21} b_{22}^{2} & b_{22}^{3}
\end{array}\right]\right) .
\end{aligned}
$$

\section{PROPOSED METHOD}

The diagram of the proposed algorithm is shown in Fig. 2. Given a reference image and a target image to be registered, the proposed algorithm starts by constructing the local phase coherence maps for both images. Each image is decomposed into multi-orientation 3scale sub-bands using the complex version of the steerable pyramid decomposition $[13,7]$. Based on Eq. (6), we define a measure of

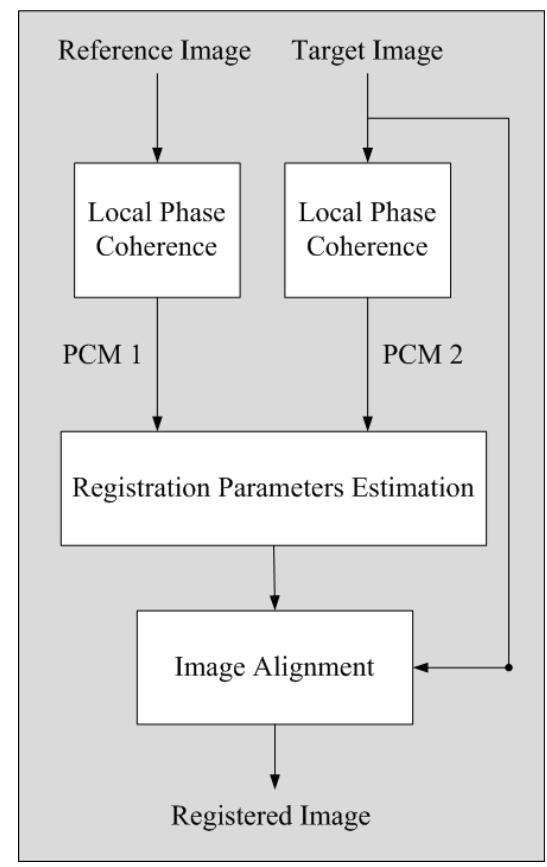

Fig. 2. Overall structure of the proposed algorithm.

local phase coherence measure as follows:

$$
P C M_{i}=\frac{\sum_{o}\left|c_{i}\right| \cos \left(\Phi\left(\left\{c_{i}\right\}\right)-\widehat{\Phi}\left(\left\{c_{i}\right\}\right)\right)}{\sum_{o}\left|c_{i}\right|+K},
$$

where $K$ is a positive constant to avoid instability at small energy regions, and its value depends on the image dynamic range and the number of orientations used; $\Phi\left(\left\{c_{i}\right\}\right)$ is the phase of each coefficient in the finest subband; and $\widehat{\Phi}\left(\left\{c_{i}\right\}\right)$ is the predicted phase using Eq. (6). This measure achieves the maximal value (which is capped by 1 ) when the phase prediction (and thus local phase coherence) is perfect. This is expected to occur in the vicinity of sharp image features. The measure is weighted by the magnitudes of the coefficients over orientations, so that the orientations that contains more energy are given higher weights. Figure 3 shows two synthetic images and their local phase coherence maps. As expected, high phase coherence values are achieved at all the sharp features, regardless of feature types (edges or lines), contrast or luminance strength, and the polarity of foreground-background intensities.

The second phase of the proposed algorithm is to estimate the registration parameters $\mathbf{x}$ using a novel objective function based on weighted mutual information (MI) between the two phase coherence maps. More specifically, we attempt to maximize:

$$
I(\mathbf{x})=\sum_{i} \sum_{j} w_{i j} P_{i j} \log \left(\frac{P_{i j}}{P_{i} P_{j}}\right),
$$

where $P_{i}$ and $P_{j}$ are the marginal histograms of the phase coherence maps for the reference and target images, respectively, $P_{i j}$ is the joint histogram between the two maps, and $w_{i j}$ is the weight given to each entry in the joint histogram. As a special case, the conventional MI-based approach corresponds to the case that all $w_{i j} \equiv 1$. This is problematic because the existence of partial or missing in- 

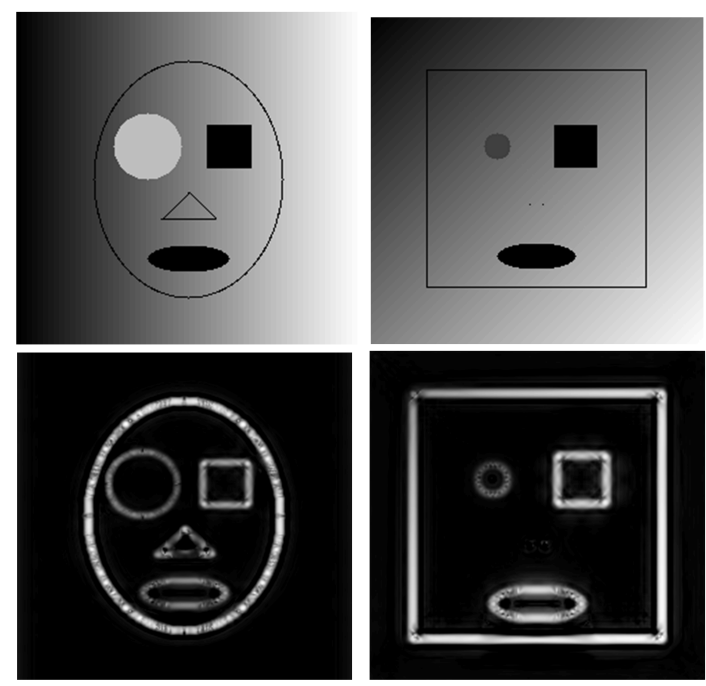

Fig. 3. Synthetic images and their corresponding local phase coherence maps.

formation between the two images may cause significant loss of correspondence in the joint histograms, and thus largely affect the estimation of the registration parameters. In our approach, we use a weighting function that assigns larger weights along the main diagonal of the joint histogram, as shown in Fig. 4. By doing so, we give less weights to outliers or points in the joint histogram with no corresponding data between the images. In other words, we put more emphasis on the points where there are possible matches between the two images. In our implementation, we allow for global translation and rotation of the images and the estimated transformation parameters $\mathbf{x}$ are obtained using a nonlinear unconstrained simplex optimization algorithm. It appears that the proposed weighting function helps the optimizer avoid being trapped in local maxima while retaining a high degree of precision in the determination of the transformation parameters. This is illustrated in Fig. 4, where the joint histogram becomes more concentrated along the main diagonal using the proposed weighted-MI approach when compared with the conventional MI-based method.

Finally, the registration parameters estimated using the local phase coherence maps are employed to transform the target image to create the registered image.

\section{EXPERIMENTAL RESULTS}

In this section, the proposed algorithm is evaluated with three sets of experiments using both synthetic images (Test Data 1, TD1, e.g., Fig. 3) and MRI (PD-MRI and T1-MRI) brain images obtained from the National Library of Medicine Visible Human Project (Test Data 2, TD2, e.g., Fig. 7) [14]. The goal of the first experiment is to show the advantage of weighted MI over conventional MI in the case of partial and missing data. To make a fair comparison, the conventional MI approach being compared was implemented using exactly the same way as the proposed algorithm, except that the weights $w_{i j}$ in Eq. (8) were fixed to be 1. Two synthetic face images from TD1 are illustrated in Fig. 3. It can be observed that these two images have only two common objects (the mouth and the right eye), and all the other components are either different or completely missing

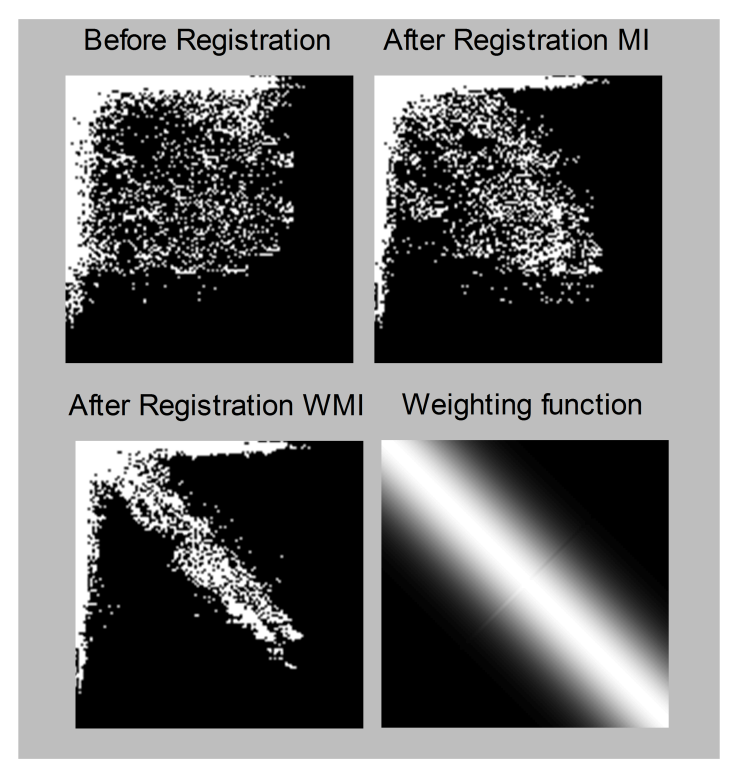

Fig. 4. Joint histograms of local phase coherence maps before and after MI and weighted-MI registration, along with the weighting function.

Table 1. RMS error comparison of MI and WMI methods

\begin{tabular}{ccc}
\hline \hline Method & Weighted MI & MI \\
& $x, y, \theta$ & $x, y, \theta$ \\
\hline TD1 & $0.0796,0.0721,0.151$ & $1.645,1.325,2.397$ \\
TD2 & $0.2181,0.2036,0.152$ & $5.049,4.474,3.800$ \\
\hline
\end{tabular}

from one of the images. The target image was altered by 11 sets of rigid transformations (translation and rotation), where the transformations range from -20 to 16 pixel shift along x-direction, -15 to 16 pixel shift along y-direction, and from 3 to 10 degrees of counterclockwise rotation around the center of the image. Similar transformations were applied to the PD-MRI and T1-MRI brain images in TD2, where the transformation parameters ranged from -24 to 20 pixel shift in $\mathrm{x}$-direction, -30 to 18 pixel shift in $\mathrm{y}$-direction, and from -10 to 10 degrees of rotation around the image center. To evaluate registration accuracy, root mean squared (RMS) error between the ground truth transformation and the estimated registration parameters is calculated. Table I compares the average RMS results between conventional MI and the proposed weighted MI methods. It can be seen that for both test data sets, the proposed method have significantly smaller RMS error, demonstrating the effectiveness of the weighted MI approach.

The purpose of the second experiment is to evaluate the robustness of the proposed algorithm in the presence of noise. Both the reference and target images are corrupted by additive independent white Gaussian noise at a wide range of noise levels. An example is given in Fig. 5, where it can be observed that the proposed method provides us with accurate registration result, regardless of the heavy noise. The RMS performance comparison of the proposed algorithm and the conventional MI method is shown in Fig. 6. It can be seen that the proposed method performs better at almost all noise levels and the resulting RMS values are quite small with the existence of very heavy noise, demonstrating strong robustness against noise. We 


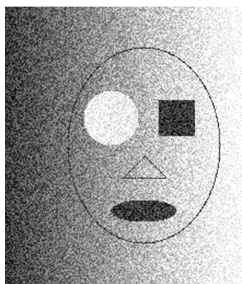

(a)

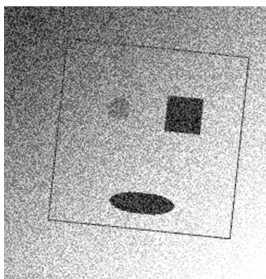

(b)

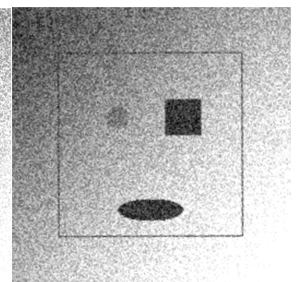

(c)

Fig. 5. (a) Noisy reference image; (b) Noisy target image; (c) Registered image.

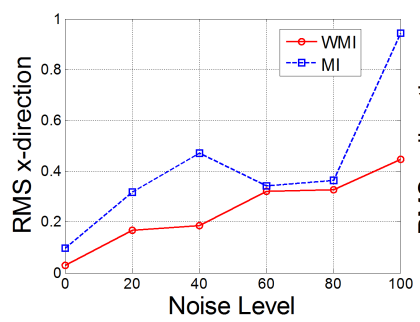

(a)

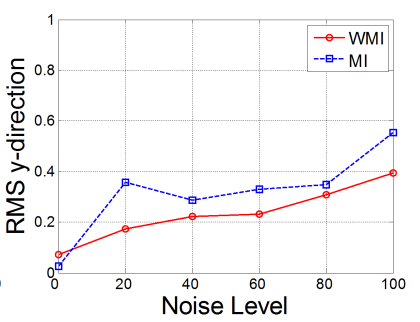

(b)

Fig. 6. Comparison of RMS estimation error in the (a) $x$-direction and (b) $y$-direction against different noise levels.

believe that such noise-robustness is a result of the stability of the proposed local phase coherence measure as of Eq. (7), which creates small values for noise patterns (because of their phase randomness and relatively low magnitudes).

The third experiment is designed to show the performance of the proposed algorithm in the case of significant missing information between the images. As shown in Fig. 7, the missing data is simulated by cutting half of the target T1-MRI image, which is to be registered with a reference PD-MRI image. It can be seen that the proposed algorithm correctly realigns the target image into the right position, regardless of the large amount of missing data, the apparent intensity variations between different imaging modalities, as well as the significant translation and rotation.

\section{CONCLUSIONS}

We propose a new method for multi-sensor image registration, which is based on two key ideas. The first is the introduction of a local phase coherence measure as a new saliency feature, which is simultaneously insensitive to luminance/contrast variations and noise contamination. The second idea is the use of a weighted mutual information-based objective function in searching the optimal registration parameters, so that more weights are given to the points that are likely to find correspondence between the images being registered. The proposed method demonstrates good performance with both synthetic and MRI brain images in the cases of significant intensity and contrast variations, noise contamination, and partial and missing data between the images.

\section{REFERENCES}

[1] F. Maes, A. Collignon, D. Vandermeulen, G. Marchal, and P. Suetens, "Multimodality image registration by maximiza-

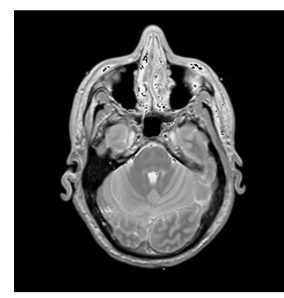

(a)

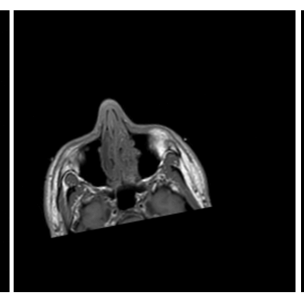

(b)

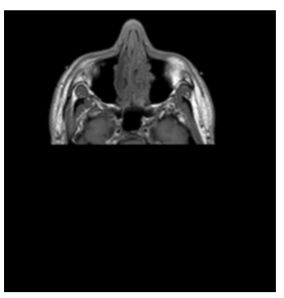

(c)
Fig. 7. (a) PD-MRI reference image; (b) T1-MRI target image with missing data; (c) Registered image.

tion of mutual information," IEEE Trans. Medical Imaging, vol. 16, pp. 187-198, 1997.

[2] P. A. Viola, "Alignment by maximization of mutual information," Int'l J Computer Vision, pp. 16-23, 1995.

[3] J. B. A. Maintz, P. A. van den Elsen, and M. A. Viergever, "Comparison of edge-based and ridge-based registration of CT and MR brain images," Medical Image Analysis, vol. 1, no. 2, pp. 151-161, 1996.

[4] Z. Wang and E. P. Simoncelli, "Local phase coherence and the perception of blur," in Adv. Neural Information Processing Systems (NIPSO3), pp. 786-792, 2004.

[5] D. J. Fleet and A. D. Jepson, "Computation of component image velocity from local phase information," Int'l J Computer Vision, , no. 5, pp. 77-104, 1990.

[6] D. J. Fleet, "Phase-based disparity measurement," CVGIP: Image Understanding, , no. 53, pp. 198-210, 1991.

[7] J. Portilla and E. P. Simoncelli, "A parametric texture model based on joint statistics of complex wavelet coefficients," Int'l J Computer Vision, vol. 40, pp. 49-71, 2000.

[8] J. Daugman, "Statistical richness of visual phase information: update on recognizing persons by iris patterns," Int'l J Computer Vision, , no. 45, pp. 25-38, 2001.

[9] M. Mellor and M. Brady, "Phase mutual information as a similarity measure for registration," Medical Image Analysis, , no. 9, pp. 330-343, 2005.

[10] D. J. Fleet and A. D. Jepson, "Stability of phase information," IEEE Trans. Pattern Anal. Mach. Intell., vol. 15, no. 12, pp. 1253-1268, 1993.

[11] A. Wong and J. Orchard, "Robust multimodal registration using local phase-coherence representations," Journal of Signal Processing Systems: Special Issue on Biomedical Imaging, vol. 54, no. 1-3, pp. 89-100, 2008.

[12] P. Kovesi, "Phase congruency: A low-level image invariant," Psych. Research, vol. 64, pp. 136-148, 2000.

[13] E. P. Simoncelli, W. T. Freeman, E. H. Adelson, and D. J. Heeger, "Shiftable multi-scale transforms," IEEE Trans Information Theory, vol. 38, no. 2, pp. 587-607, Mar 1992.

[14] National Library of Medicine, National Institute of Health, "Visible human project," http://www.nlm.nih.gov/ research/visible/visible_human.html. 